\title{
ARTICLE \\ Microanatomical dissection of human intestinal T-cell immunity reveals site-specific changes in gut-associated lymphoid tissues over life
}

\author{
Takashi Senda ${ }^{1,2}$, Pranay Dogra ${ }^{1,3}$, Tomer Granot ${ }^{1,3,5}$, Kazuhiro Furuhashi ${ }^{1}$, Mark E. Snyder ${ }^{1,3}$, Dustin J. Carpenter ${ }^{1,2}$, Peter A. Szabo ${ }^{1,3}$,
} Puspa Thapa ${ }^{1,3}$, Michelle Miron ${ }^{1,4}$ and Donna L. Farber ${ }^{1,2,4}$

Defining adaptive immunity with the complex structures of the human gastrointestinal (Gl) tract over life is essential for understanding immune responses to ingested antigens, commensal and pathogenic microorganisms, and dysfunctions in disease. We present here an analysis of lymphocyte localization and T cell subset composition across the human GI tract including mucosal sites (jejunum, ileum, colon), gut-associated lymphoid tissues (isolated lymphoid follicles (ILFs), Peyer's patches (PPs), appendix), and mesenteric lymph nodes (MLNs) from a total of 68 donors spanning eight decades of life. In pediatric donors, ILFs and PP containing naïve $T$ cells and regulatory $T$ cells (Tregs) are prevalent in the jejunum and ileum, respectively; these decline in frequency with age, contrasting stable frequencies of ILFs and T cell subsets in the colon. In the mucosa, tissue resident memory T cells develop during childhood, and persist in high frequencies into advanced ages, while T cell composition changes with age in GALT and MLN. These spatial and temporal features of human intestinal T cell immunity define signatures that can be used to train predictive machine learning algorithms. Our findings demonstrate an anatomic basis for age-associated alterations in immune responses, and establish a quantitative baseline for intestinal immunity to define disease pathologies.

Mucosal Immunology (2019) 12:378-389; https://doi.org/10.1038/s41385-018-0110-8

\section{INTRODUCTION}

The intestinal immune system has evolved to generate robust responses against pathogens while suppressing immune activation to non-pathogenic antigens, such as food and commensal flora. However, this balance becomes dysregulated in certain diseases of the gastrointestinal (GI) tract, which show a strong association with age and specific location. For example, sensitivity to ingested antigens (i.e. food allergies) and oral tolerance occur more readily during early life, ${ }^{1}$ inflammatory bowel disease (IBD) can involve either the small or large intestine and also occurs more often among the pediatric and young adult population, ${ }^{2}$ and conversely, cancer of the intestine is typically confined to the colon and occurs more often in late middle age. ${ }^{3}$ These age- and site-specific pathologies suggest spatial and temporal regulation of intestinal immunity, though in humans, the processes by which immune cell populations interact with and take up residence within the Gl tract and gut-associated lymphoid tissues (GALT) are not known.

Immunity in the $\mathrm{Gl}$ tract consists of the intestinal epithelial barrier, populated with intraepithelial lymphocytes (IELs), the lamina propria (LP) populated with lymphocytes and innate cells, and gut-associated lymphoid tissue (GALT) comprising Peyer's patches (PPs), isolated lymphoid follicles (ILF), the appendix, and multiple mesenteric lymph nodes (mLNs) draining different regions of the intestinal tract. ${ }^{4,5}$ The development of intestinal T cell immunity has been largely studied in mice, where PPs have been shown to develop In utero needs to be italicized. along with mLNs. ${ }^{4}$ In mice ILFs develop within LP after birth during bacterial colonization of the gut ${ }^{6}$, while in humans ILFs are present at birth and play roles in priming early intestinal immune responses. ${ }^{7}$ How T cell subsets differentiate and populate ILFs and PPs in later life in mice is not well characterized. ${ }^{8}$ Developmental studies of the human intestinal immune system have been limited by the difficulty in obtaining samples of healthy samples from different parts of the Gl tract. ${ }^{7,9,10}$ The development of GALT and lymphoid follicles within the intestines remains largely undefined in humans.

$T$ lymphocytes play a major role in maintaining protective immunity, memory, tolerance, and tissue homeostasis in the $\mathrm{Gl}$ tract. The major $\mathrm{T}$ cell subsets populating mouse and human intestines are memory $T$ cells ${ }^{11,12}$ which arise from activated T cells after an initial antigen encounter. Intestinal memory $T$ cells are predominantly non-circulating, tissue resident memory $T$ cells (TRM) exhibiting distinct phenotypes such as upregulation of CD69 and CD103 expression for tissue retention. ${ }^{1,14}$ The timing and pathways for intestinal TRM establishment have not been defined for mice or humans. $\mathrm{CD4}^{+}$regulatory $\mathrm{T}$ cells (Tregs) are another key subset for intestinal immunity ${ }_{i}^{15}$ Tregs prevent and cure intestinal inflammation in mouse models of inflammatory

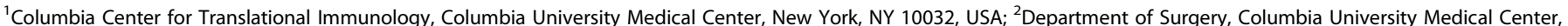

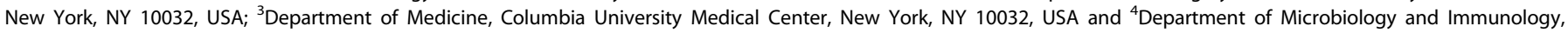
Columbia University Medical Center, New York, NY 10032, USA

Correspondence: Donna L. Farber (df2396@cumc.columbia.edu)

${ }^{5}$ Present address: Compugen, San Francisco, CA, USA

Received: 2 July 2018 Accepted: 30 October 2018

Published online: 6 December 2018 
bowel disease (IBD). ${ }^{16,17}$ While there is a significant frequency of mouse Treg in the GI tract and GALT throughout the lifespan of the mouse, ${ }^{18}$ it is unknown whether this applies to human intestines. The relative levels and localization of $T$ cell subsets, including memory T cells, TRM, and Tregs, in the human Gl tract at different life stages have not been reported.

Here, we used our unique human tissue resource where we obtain multiple mucosal and lymphoid tissues from organ donors, ${ }^{11,19,20}$ for in situ characterization of adaptive immunity in the human intestinal tract across all anatomic regions and ages. Using immunofluorescence imaging and flow cytometry, we identified tissue- and age-specific changes in the presence of lymphoid follicles and in $T$ cell composition within intestinal mucosal sites and associated lymphoid tissues. We identified a large number of ILFs containing naïve $T$ cells and Tregs in the jejunum, PP and LP in early life that decline with age showing sitespecific kinetics; the fastest decline occurs in the jejunum, followed by PP within the ileum, while the colon retains similar numbers of ILF at all ages. Mucosal sites in donors of all ages are stably populated with a predominant TRM population from childhood ( $<5$ years) until advanced ages ( $>85$ years). Multidimensional analysis suggests that aging plays a significant role in shaping the immune parameters of the GALT and $\mathrm{mLN}$, compared to mucosal tissues; individual contributions of age and tissue site are further demonstrated using a predictive machine learning algorithm. Our findings identify age-related cellular and structural changes in intestinal T cell immunity, and provide a new baseline for defining immune pathologies in the intestines over space and time.

\section{RESULTS}

Samples for analysis of lymphocytes in human intestinal mucosal sites, GALT and associated LN

We have established a resource to obtain multiple lymphoid and mucosal tissues from research-consented human organ donors through a collaboration with the LiveOnNY as previously described ${ }^{11,19-23}$. We characterized mucosal intestinal tissues, GALT and lymphoid sites (Fig. 1a) from donors across all ages of life (4 months-87 years) combining fluorescence imaging, flow cytometry, and multiparameter analysis platforms (Fig. 1b). Unique aspects of our sampling include the simultaneous acquisition of intestinal tissues, GALT, and LN from the same donors, including PPs, present as macroscopically visible intestinal lymphoid structures mostly concentrated in the terminal ileum. ${ }^{24}$ We surgically excised PPs from the proximal and distal ileum, and $\mathrm{mLN}$ draining different sites based on their location (Fig. 1a).

Tissues were acquired from 68 individual donors (Supplementary Table 1), aged 4 months-87 years; age-matched samples were used for imaging (median age 32.6 years, 50\% male) and flow cytometry (median age 42.2 years, 58\% male) (Fig. 1b). Donors were classified into three life stages: young ( $0-24$ years), middle (25-49 years), and older (50+ years) to investigate the dynamics of intestinal immune parameters over human life.

\section{ILF show age-related changes in distribution and lymphocyte composition}

The distribution and organization of lymphocytes in human small and large intestine was investigated by immunofluorescence staining for CD4, CD8, CD45RA, and DAPI allowing visualization of T cells and demarcation of B cell follicles (Supplementary Figure 1a, b). ${ }^{25}$ ILF can be visualized within intestinal sites as regions of lymphoid aggregates with a central B cell cluster (red) surrounded by a $\mathrm{T}$ cell zone (green) (Fig. 2a), consistent with previous studies. ${ }^{4,26}$ In jejunum and colon, ILFs comprised of one or more B cell follicles surrounded by $T$ cells were located at the base of the LP, near the submucosal (SM) region (Fig. 2a). In the jejunum of younger donors, particularly $<2$ years of age, ILFs consisted of multiple follicular regions, while in older children and adults, ILFs were present as isolated follicles (Fig. 2a, left column). Although lymphoid follicles are retained in the PPs of ileum throughout all stages of life (Fig. 2a, middle, Supplementary Figure 1c, d), they were more numerous in infants and children, with greater $T$ cell density in the interfollicular region compared to PP in middle and older donors. By contrast, ILFs in the colon consisted mainly of single B-cell follicles surrounded by $\mathrm{T}$ cells, present at all ages examined (Fig. 2a; Supplementary Figure 1e, f).

To quantify changes in follicular content with age, we calculated the number of ILFs/unit area of LP (density) and the ILF/LP area ratio (relative size). ILFs in the jejunum of younger donors were a

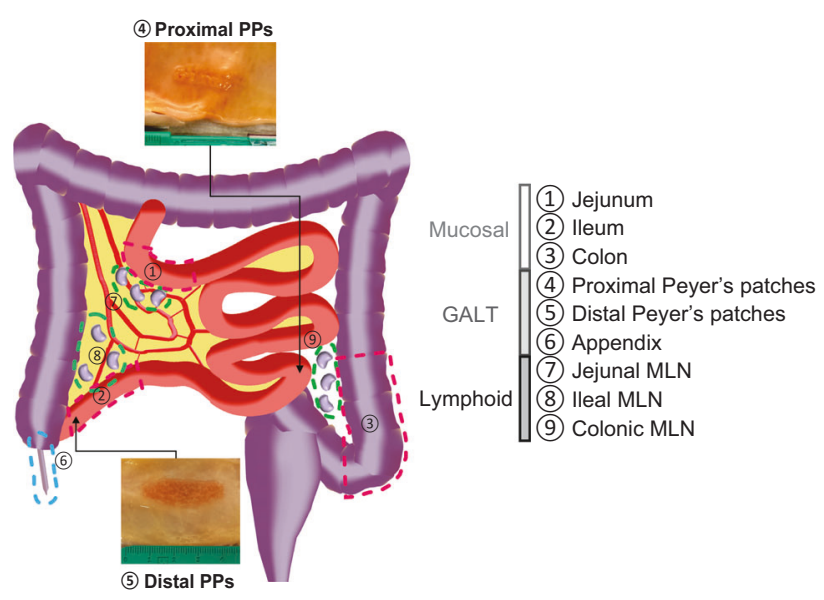

b
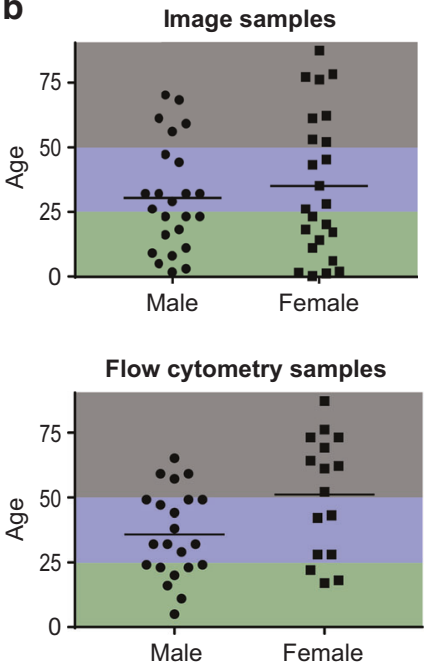

Fig. 1 Tissues and donors analyzed for this study. a Schematic diagram of the different parts of the Gl tract characterized in this study including mucosal tissues (jejunum, ileum, colon), gut-associated lymphoid tissue (GALT) consisting of Peyer's patches (PPs) and appendix, and lymphoid tissues (mesenteric lymph nodes $(\mathrm{mLN})$ draining mucosal sites) obtained from organ donors for this study. The localization of these sites is indicated on the diagram (left) by numerical designation (right). b Age range and sex of donors used for imaging (top) and flow cytometry (bottom) analyses. Age distribution of analyzed donors is grouped into three life stages: young (0-24 years, green), middle (25-49, blue), and older (50+ years, gray) 

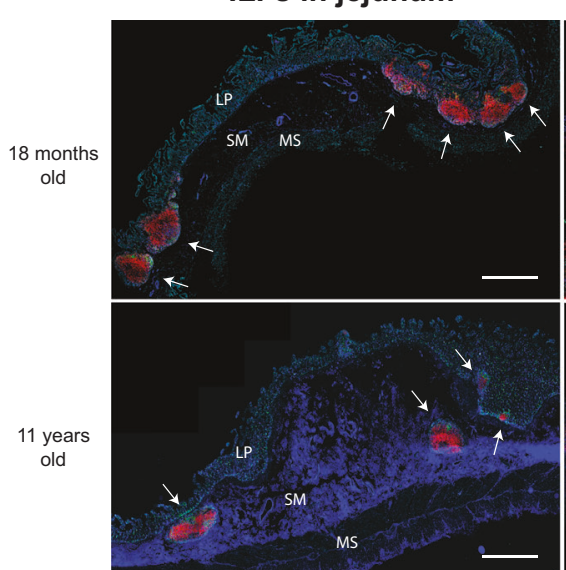

17 years

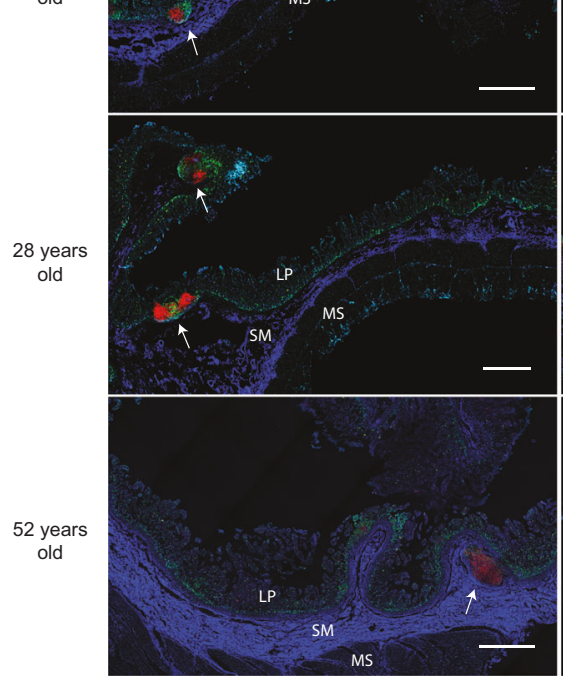

b

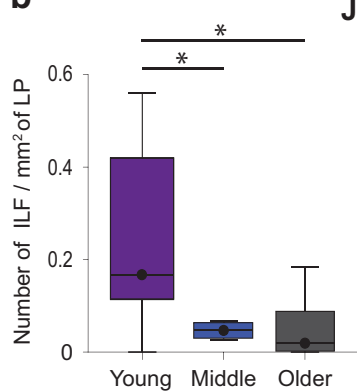

Jejunum

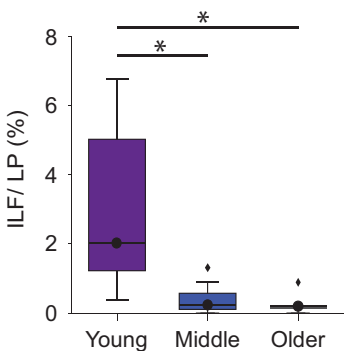

PPs in ileum
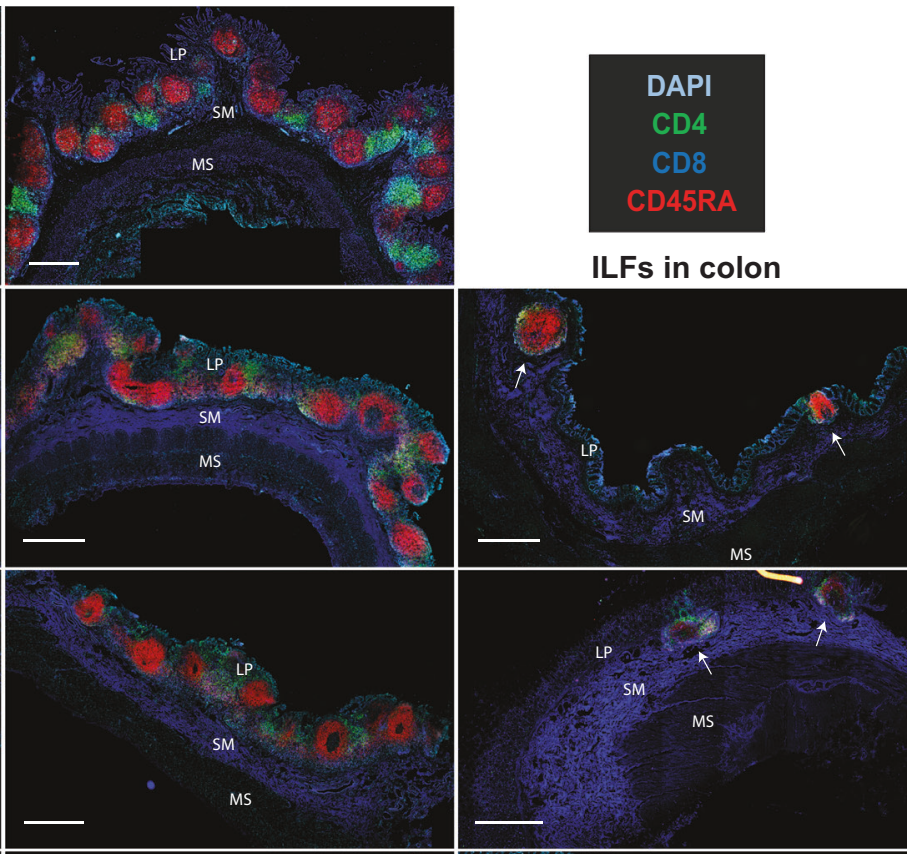

MS
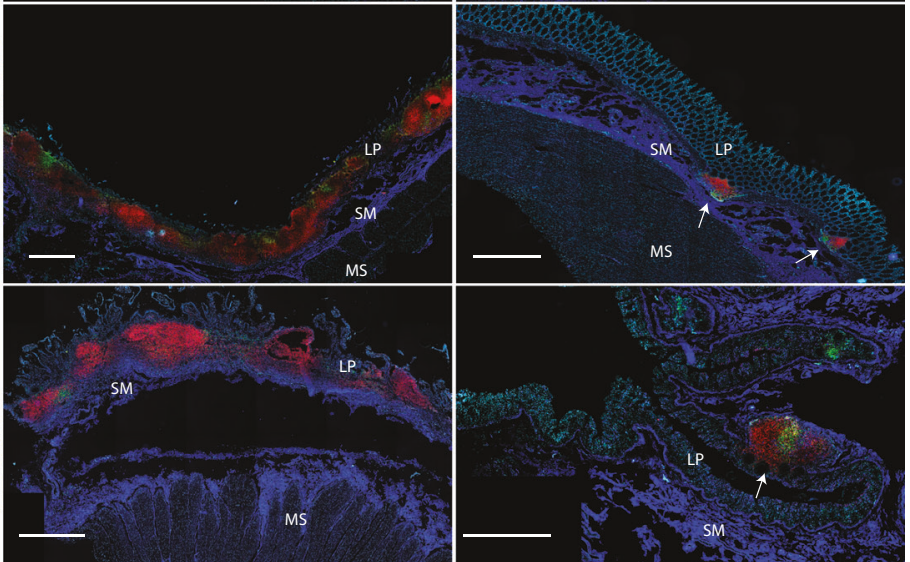

C

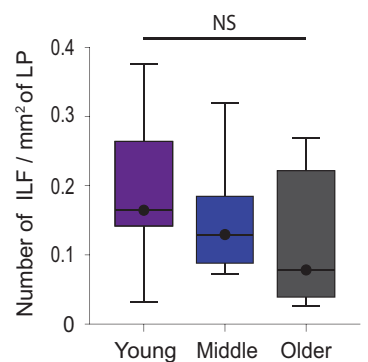

Colon

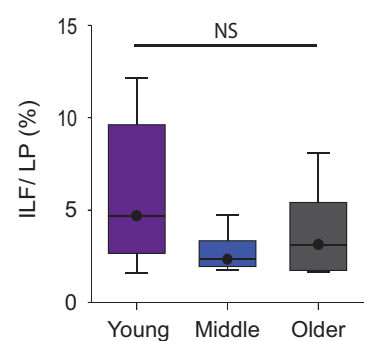

Fig. 2 Dynamic, age-associated changes in lymphoid structure in human intestines. a Visualization of lymphoid structures in jejunum (left), ileum (center), and colon (right) from donors of representative ages from early life to middle age (Donor 294, 18months; Donor 319, 11 years; Donor 321, 17 years; Donor 352, 28 years; Donor 327, 52 years). Frozen intestinal tissues were stained for expression of CD4 (green), CD8 (blue), and CD45RA (red), and DAPI (cyan) was used to visualize nucleated cells. White arrows indicate isolated lymphoid follicles (ILFs) in the jejunum and colon. All images show at $\times 10$ magnification. LP lamina propria, SM submucosa, MS muscularis. Scale bars, $1000 \mu \mathrm{m}$. $\mathbf{b}$ Quantification of ILF content and size in the jejunal ILFs in the three age groups. Graphs show the number ILFs/unit area of LP (left) and ILF/ LP area ratio (right) in the jejunum from young $(n=16$, avg. age 9 years), middle $(n=6$, avg. age 32 years) and older $(n=8$, avg. age 69 years). For each donor 2-10 samples were used for quantitation. c Quantification of ILF content and size in colonic ILFs. Graphs show ILFs/unit area of LP (left) and ILF/ LP area ratio (right) from young ( $n=7$ avg. age $=14$ years), middle $(n=7$, avg. age $=37$ years), and older $(n=4$, avg. age $=68$ years). ${ }^{*} p<0.05$, NS not significant 
increased in density and relative size compared to those of individuals in the middle and older years (Fig. 2a, b; Supplementary Figure 2a). By contrast, ILFs in the colon were maintained at similar numbers and density in all three age groups, with a trend toward a higher density of ILFs in the colon of young donors (Fig. 2c; Supplementary Figure 2a). These results show dynamic changes in the lymphoid follicle content with age particularly in the small intestine; higher numbers of lymphoid follicles are observed in younger years which diminish over time.

Age-associated changes in naïve T cell composition in the gut We investigated the $T$ cell subset composition of organized lymphoid structures in jejunum, ileum, and colon at different life stages (Fig. 3a; Supplementary Figure 1). We quantified different aspects of $\mathrm{T}$ cell subset composition and organization, including $\mathrm{CD}^{+}$and $\mathrm{CD}^{+} \mathrm{T}$ cell number in the interfollicular $\mathrm{T}$ cell region from ILFs in the jejunum, colon and PPs, and the CD4:CD8 T cell ratio in these sites for each donor. The CD4:CD8 T cell ratio within ILFs of jejunum and colon was not significantly different between the three age groups (Fig. 3b; Supplementary Figure 2b). However, we observed an increased CD4:CD8 T cell ratio in the PPs of pediatric and young donors compared to middle and older age donors (Fig. 3b; Supplementary Figure 2b), consistent with lymphoid tissues having a higher CD4:CD8 T cell ratio earlier in life. ${ }^{20}$

Coordinate staining enabled us to distinguish naïve $\mathrm{CD}^{+}$and $\mathrm{CD}{ }^{+} \mathrm{T}$ cells which express CD45RA (yellow and purple, respectively) from memory subsets which are CD45RA ${ }^{-}$(green and blue). (Although terminal effector $\mathrm{CD} 8^{+} \mathrm{T}$ cells (TEMRA) also express CD45RA, flow cytometry analysis from our previous studies and shown below revealed few TEMRA cells in LN or intestinal sites ${ }^{11,19,20}$.) Based on imaging, the frequency of naïve $\mathrm{CD}^{+}$and $\mathrm{CD}^{+} \mathrm{T}$ cells in ILFs from the jejunum, PP, and colon showed sitespecific changes with age (Fig. 3). There were higher frequencies of naïve $\mathrm{CD}^{+}$and $\mathrm{CD}^{+} \mathrm{T}$ cells in jejunal ILF in young pediatric donors, particularly in the first 2 years of life, compared to donors in middle and older years (Fig. 3c, left panel). Similarly, the PPs of young and middle-aged donors contained higher frequencies of naïve T cells, compared to PPs from donors $>50$ years (Fig. 3c, middle; Supplementary Figure 1), while colonic ILFs maintained similar naïve $T$ cell frequencies with age (Fig. 3c, right). These data demonstrate that naïve $T$ cell attrition occurs most rapidly in the jejunum, followed by PP, with memory $T$ cells predominating for the majority of life.

Intestinal TRM are established early and maintained at comparable frequencies at all life stages

We used multiparameter flow cytometry to further assess $T$ cell subset composition in the nine different intestinal sites shown in Fig. 1a. There was a progressive increase in the CD4:CD8 $T$ ratio from mucosal sites, GALT to $\mathrm{mLNs}$, with an overall lower CD4:CD8 content in younger compared to older donors (Supplementary Figure 3). The frequency of major functional T cell subsets (naïve, effector memory (TEM), central memory (TCM) and TEMRA ${ }^{27,28}$ ) was a feature of the specific intestinal location, with age-related changes only in certain sites (Fig. 4a). In mucosal sites (jejunum, ileum, and colon), TEM were the predominant subset for both $\mathrm{CD}^{+}$and $\mathrm{CD}^{+}{ }^{+}$cells across all age groups. TEMRA subsets were not significantly represented in any Gl site, while TCM cells were present in constant frequencies (20-30\%) among $\mathrm{CD} 4^{+} \mathrm{T}$ cells in GALT and $\mathrm{mLN}$ across all age groups (Fig. 4a). By contrast, GALT and $\mathrm{mLNs}$ showed differences in frequency distribution of naïve and TEM cell subsets between site and age groups (Fig. 4a). In young and middle-aged donors, there was a directional increase in naïve T cell frequency from PP to appendix (20-40\%) to $\mathrm{mLNs}$ (40-60\%) and a corresponding decrease in TEM cell frequencies along those same sites (Fig. 4b). For donors within the older age group, naïve $T$ cells comprised $<10 \%$ in GALT, similar to intestinal sites and $<20 \%$ in $\mathrm{mLNs}$ (Fig. $4 \mathrm{~b}$ ). Therefore, while the organization of lymphocytes and subset composition within GALT, mLN, and lymphoid follicles in the intestines is altered with age, the overall $T$ cell subset composition averaged across the mucosa is mostly TEM cells independent of age.

We examined the extent to which TEM cells in the Gl tract expressed markers of TRM cells including the activation marker CD69 and tissue retention integrin CD103 ${ }^{29,30}$. When gating for TEM cells, the extent of CD69 and CD103 expression was a feature of the tissue site, and did not differ with age as shown in representative flow cytometry plots from individual donors aged 5-87 years (Fig. 4c), and in compiled data from multiple sites and individuals (Fig. 4d). The major distinction in TRM markers was due to tissue site; CD69 which has been shown to delineate human $\mathrm{TRM}^{14}$ was expressed by the vast majority (80-90\%) of $\mathrm{CD}^{+}$and CD8 ${ }^{+}$TEM cells in intestines and GALT, and by a slightly lower percentage (60-80\%) of TEM cells in $\mathrm{mLN}$ sites (Fig. 4d). Similarly, the frequency of CD8 ${ }^{+}$TEM cells co-expressing CD69 and CD103 in mucosal sites (jejunum, ileum, colon, 60-80\%) was much higher compared to TEM in GALT (40-60\%) and $\mathrm{mLN}(10-20 \%)$, while CD103 was expressed only by a low frequency of intestinal $\mathrm{CD} 69^{+} \mathrm{CD} 4^{+}$TEM cells (Fig. $4 \mathrm{~d}$ ). We also investigated the localization of TRM within the jejunum by imaging analysis revealing that $\mathrm{CD} 9^{+}$or $\mathrm{CD}_{103^{+}} \mathrm{T}$ cells are predominantly present in the LP area, with only low frequencies of TRM within the ILFs (Supplementary Figure 4). These data reveal novel features of human TRM across the GI tract: first, that TRM cells are formed early within intestinal sites and may be retained there long term, and second, the extent of TRM establishment is a function of the tissue site.

Localization of Intestinal Tregs in GALT with highest frequencies during childhood

We also used imaging and flow cytometry to investigate the localization of Tregs in the human Gl tract and age-related effects. Immunofluorescence staining of CD4 and Foxp3 shows Tregs present as aggregates within ILFs of jejunum and colon, and PPs within the ileum (Fig. 5a), but only sparsely and rarely distributed within the LP and intraepithelial sites (not shown). For quantitation of Treg frequency, we further stratified younger donors into pediatric (0-10 years) and youth (11-24 years) and found a significant increase in Treg frequency within ILFs in the jejunum and PP of pediatric donors compared to younger and older adults (Fig. 5b, c). In contrast, there was no significant difference in Treg frequency within ILFs of the colon across all age groups (Fig. 5b; Supplementary Figure 2c). We further investigated Treg frequency in these sites by flow cytometric analysis using a triple gating strategy. ${ }^{31}$ In intestinal sites, we detected a low $(<2 \%)$ Treg frequency that did not change with age (Fig. $5 \mathrm{~d}$ ), consistent with our imaging data. In the GALT and $\mathrm{mLNs}$, however, Treg frequency was highest in younger donors (5-10\%) and significantly reduced in middle and older-aged donors (1-3\%; Fig. 5c, d). Moreover, the frequency of naïve (CD45RA ${ }^{+}$) Tregs in the colon, PPs, and mLNs was higher in younger compared to older donors (Fig. $5 \mathrm{~d}$ ). These data indicate a higher Treg content in the Gl tract in younger individuals, and particularly in children, which declines with age, albeit at different rates in GALT and lymphoid sites.

\section{Quantifying effects of age and sex in regional intestinal} immunity

To visualize the relatedness between different regions of the human intestine based on the immune parameters measured, we performed a $t$-distributed stochastic neighbor embedding (t-SNE) analysis using flow cytometry data collected from our donors for 14 immune parameters as described in the previous sections (see Methods). The first two t-SNE components are visualized showing data from all the sites with each point representing one donor and one site shaded according to the donor age (Fig. 6a, left) and for each individual site shown at right (Fig. 6a, right). We delineated 
a

$\mathrm{CD}^{+} \mathrm{T}$ cells

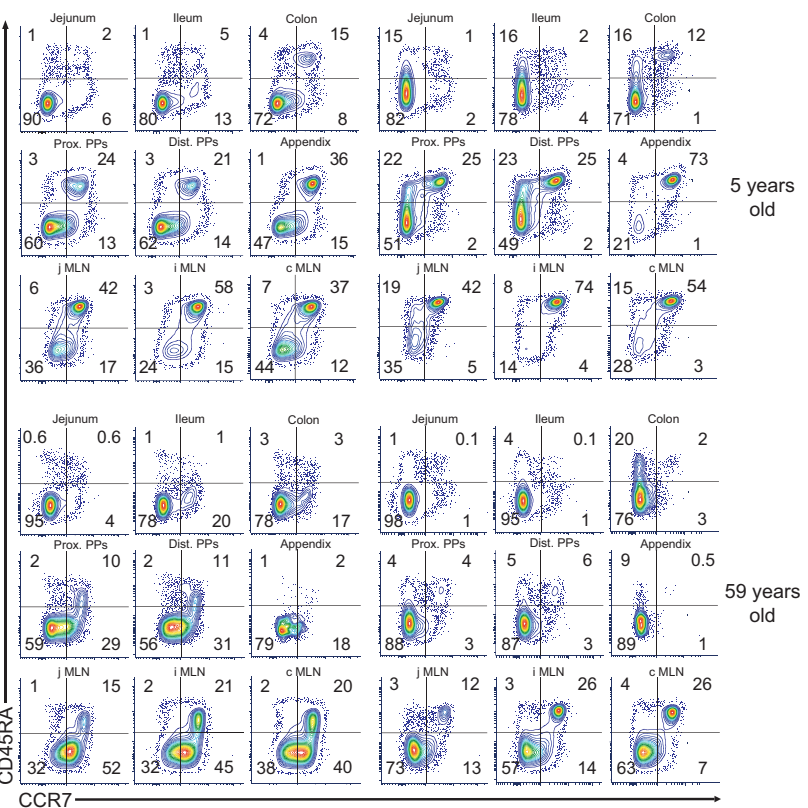

b
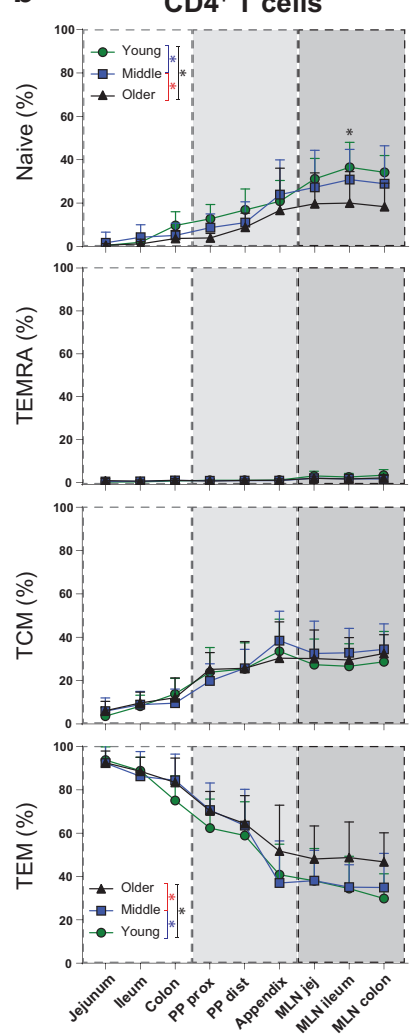

$\mathrm{CD}^{+} \mathrm{T}$ cells

D8 ${ }^{+} \mathrm{T}$ cells
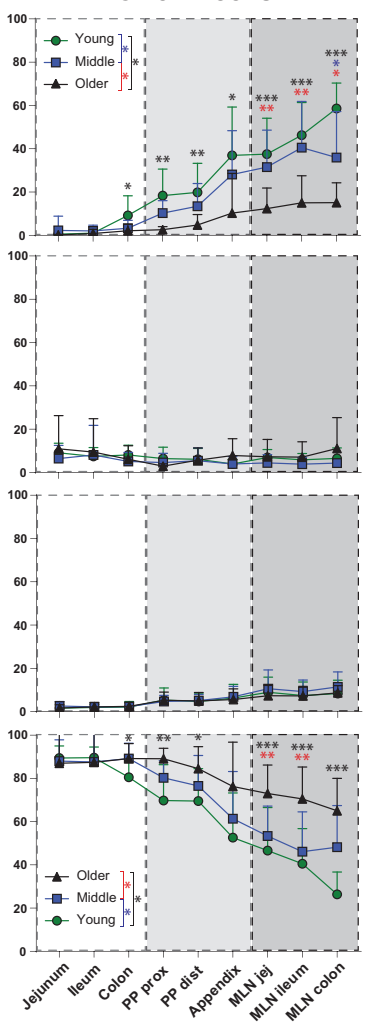

C
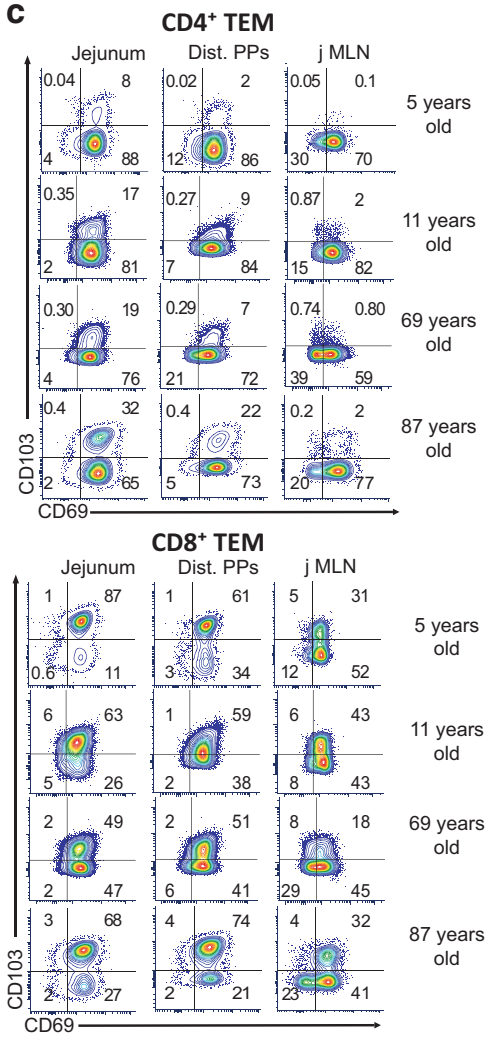

d
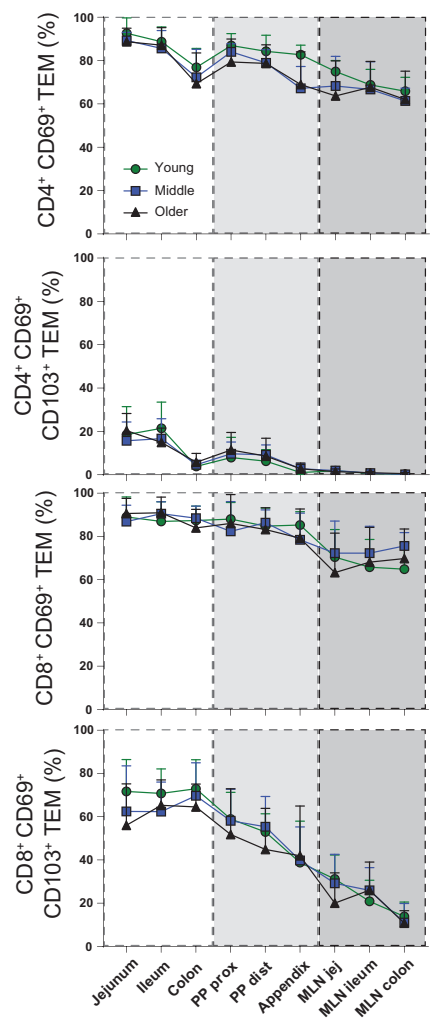

three basic clusters of samples based on k-means clustering analysis of these data (Fig. 6). Overall, mucosal sites cluster separately from GALT and $\mathrm{mLN}$ sites for all donors based on the measured lymphocyte parameters (Fig. 6a, left; Supplementary
Figure 6a). For the appendix, younger donors seem to cluster separately from older ones based on the t-SNEx component, while no such separation based on age was observed for mucosal sites (Fig. 6a, right). Interestingly, the PPs occupied an intermediate 
Fig. 4 Tissue distribution of major T cell subsets in the human intestinal tract with age. a Gating strategy used to identify $T$ cell subsets based on CD45RA and CCR7 expression defining: CD45RA ${ }^{+} / \mathrm{CCR7}^{+}$(Naive), CD45RA ${ }^{-} / \mathrm{CCR7}^{+}$(Central Memory or TCM), $\mathrm{CD}^{2} 5 \mathrm{RA}^{-} / \mathrm{CCR7}^{-}$(effector $^{-}$ memory or TEM), CD45RA ${ }^{+} / C C R 7^{-}$(Terminal effector or TEMRA). Flow cytometry plots of CD45RA and CCR7 expression by CD4 ${ }^{+}$and CD8 ${ }^{+}$ T cells in human intestinal tissues from a representative younger (Donor 339, 5 years) and older (Donor 330, 59 years) individual. b Mean frequency $\left( \pm\right.$ SEM) of Naïve, TEMRA, TCM, TEM cells expressed as a percent of $C D 4^{+}$(left) or CD8 ${ }^{+}$(right) T cells in lamina propria, GALT, and $\mathrm{mLNs}$ from young (green; $n=11$ ), middle (blue; $n=13$ ), and older (gray; $n=13$ ) donors. Statistical significance comparing young to older (black*), young to middle (blue*), and middle to older ( red $^{*}$ ), indicated as ${ }^{*} p<0.05 ;{ }^{*} p<0.01,{ }^{* *} p<0.001$. c Representative flow cytometry plots of CD69 and CD103 expression by CD4 ${ }^{+}$and CD8 ${ }^{+}$TEM cells from 4 individuals (Donors 339, 5 years; 351,11 years; 349,69 years; 364,87 years) in the indicated mucosal, GALT, and lymphoid sites. $\mathbf{d}$ Mean frequencies \pm s.e.m. of CD4 ${ }^{+} \mathrm{CD} 9^{+}$(top), $\mathrm{CD}^{+} \mathrm{CD} 69^{+} \mathrm{CD} 103^{+}(\mathrm{second}$ row from the top), $\mathrm{CD}^{+} \mathrm{CD}^{+} 9^{+}$(third row from the top), and $\mathrm{CD}^{+} \mathrm{CD}^{+} 9^{+} \mathrm{CD} 103^{+}$(bottom) TEM cells from young (green; $n=11$ ), middle (blue; $n$ $=13$ ), and older (gray; $n=13$ ) donors. No significant differences were observed between age groups

position in the clusters (Fig. 6a, left). In donors where PP resembled $\mathrm{mLN}$, the PPs appear to partition by age, whereas for donors where PPs resembled mucosal tissue, no partitioning by age was observed. (Fig. 6a, right). These results suggest that tissue niche plays a prominent role in shaping the immune phenotype of the human intestine. Additionally, aging influences immune parameters in MLN and GALT to a greater extent than in mucosal sites, with effects on PP specific to the individual.

To further investigate impacts of age on the immune composition of the Gl tract we carried out multivariate analysis adjusting for both sex and age. After adjusting for age, the majority of immune parameters did not differ between males and females except that the frequency of Tregs and CD4 ${ }^{+}$TRM in the ileum, GALT, and certain MLN sites was higher in males compared to females (Fig. 6b, c). Aging, however, affected a greater number of immunological parameters across the human intestine. Adjusting for sex, we observed an inverse correlation between age and naïve $\mathrm{CD}^{+}{ }^{+} \mathrm{T}$ cell frequency in the colon, and between age and naïve $\mathrm{CD}^{+} \mathrm{T}$ cell frequency in the colon and $\mathrm{mLNs}$ from all the sites across the intestine. Conversely, $\mathrm{CD}^{+}$and $\mathrm{CD} 8^{+} \mathrm{TEM}$ and $\mathrm{CD}^{+}$TEMRA frequency increased with age in all $\mathrm{mLN}$ sites and the colon (Fig. 6b, d). Together, these results show differential impact of age and sex on specific sites within the GI tract; GALT and lymphoid sites are more affected by age, while mucosal sites exhibit some sex-specific effects.

Machine learning algorithms can predict the age and tissue site of healthy human donor GI tract

There is evidence showing an association between certain pathologies of the Gl tract, age, and intestinal site., 2,3,32 Our intestine immune cell dataset can serve to establish a baseline from which to evaluate pathologies of the GI tract. As a first step towards this goal we asked whether the immune parameters measured here are sufficient to identify the proper age and correct anatomical site of the intestine and therefore serve as a key reference point. Several of immune parameters measured across different regions of the intestine exhibit high degrees of correlation (Supplementary Figure 5). We hypothesized that T cell composition as determined here could itself be predictive of the age and tissue site in the human GI tract. We therefore applied a custom Python script implementing supervised Random forest classifier algorithm on our dataset. Machine learning has been implemented in a variety of diagnostic settings from classifying cancer types, to predicting the risk of cardiac disease and cancer outcomes. ${ }^{33-35}$ We used the aggregated dataset from all donors as input into a machine learning algorithm, and then asked whether the "trained" algorithm could accurately predict the tissue site and/or age of an unknown sample. Running the full model with 16 immunological parameters resulted in an accuracy score of 0.88 for age group prediction and 0.87 for tissue site prediction; excluding the parameters with low importance (Fig. 7a, Supplementary Figure $6 \mathrm{~b}, \mathrm{c}$ ) resulted in similar accuracy scores for both models (Fig. 7b, c).

Results of the age group prediction model summarized as confusion matrix in Fig. 7c shows that middle and young age groups donors could be classified with high accuracy; however, one of the older donors (59 years) was misclassified as middle aged due to a higher frequency of $\mathrm{CD} 4^{+}, \mathrm{CD} 8^{+} \mathrm{TCM}$, and lower frequency of $\mathrm{CD}^{2} 5 \mathrm{RA}^{+}$Tregs compared to other older donors in the cohort. Similarly, for tissue site prediction, we were able to achieve perfect classification for GALT and MLN, although several mucosal samples were misclassified (Fig. 7c), based on variations in TRM frequencies. Our results serve as proof of concept to use T cell subset composition to predict the tissue site and age of an unknown Gl sample, and conversely to define specific immune alterations of samples from a known site and age.

\section{DISCUSSION}

Defining adaptive immunity in the intestine and how it changes over human life is a necessary foundation for understanding immune-related intestinal dysfunctions and diseases. However, most our knowledge of intestinal immunity derives from animal models. Here, we analyzed $\mathrm{T}$ cell maturation and localization across the human $\mathrm{Gl}$ tract in nine different sites including small and large intestines, GALT, and associated mLN from 68 organ donors spanning nine decades of life. Our results provide new insights into the spatial and temporal dynamics of the adaptive immune system in human intestinal immunity that cannot be extrapolated from animal models or isolated biopsy samples. The small intestine of young donors contains numerous large ILFs in the jejunum and PP containing high frequencies of naïve $T$ cells and Tregs, which decline with age with site-specific kinetics; jejunum shows the most rapid decline at early ages followed by ileum, and colon maintaining ILFs over life. Within the mucosa, however, TRM are established in childhood and their frequency and distribution is stable into advanced ages, while GALT and MLN show gradual changes in T cell subset composition over life. These site- and age-specific features of human intestinal immunity are highly consistent between individuals and predictable using machine learning approaches. Together, these findings establish a new quantitative baseline for human intestinal immunity to define and understand disease pathologies.

The full spectrum of intestinal adaptive immunity involves T cells within mucosal sites, GALT, and MLN draining different intestinal regions. Previous studies on T cells isolated from human intestine have been limited to tissues surgically excised or biopsy samples from the patients who had malignancy or IBD. ${ }^{10,36}$ Our longstanding collaboration with LiveOnNY allows us access to multiple lymphoid, mucosal, and peripheral tissue sites from previously healthy donors. ${ }^{11,19}$ This unique resource has allowed us to characterize global and regional changes in the human immune system on the cellular and molecular level. ${ }^{14,20,22,23}$ However, in these previous studies, cells were isolated and characterized from digested tissues, while in this study, we investigated the cellular localization and organization in situ using imaging compared to the cellular analysis from tissue digestion. We demonstrate dynamic, age-associated changes in the organization and composition of lymphocytes in ILFs and PPs within the small intestines in the context of stable T cell subset composition 

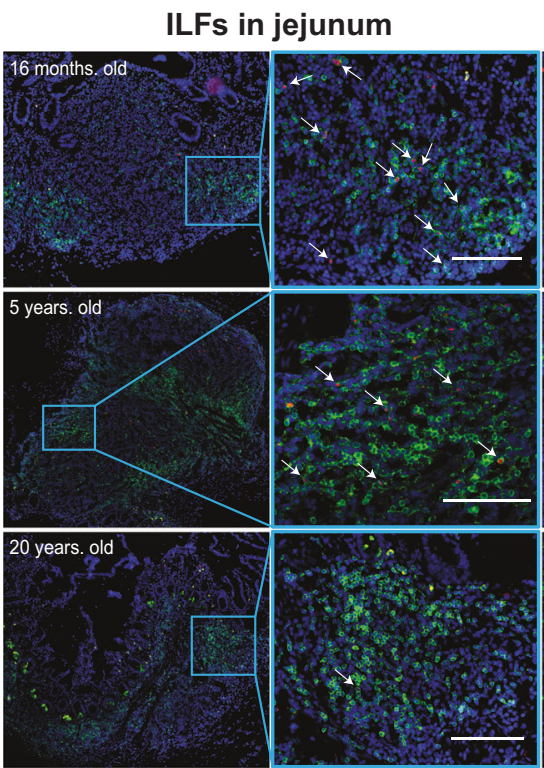

b

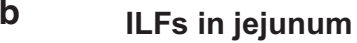

PP
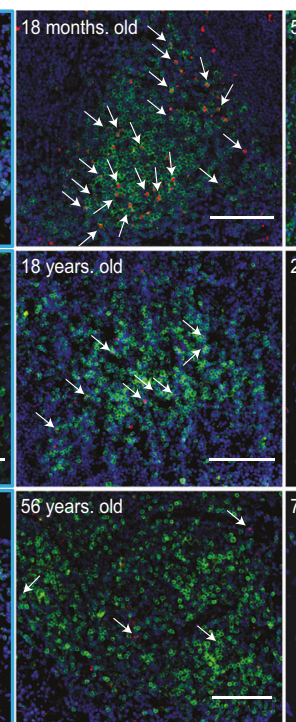

PPs in ileum
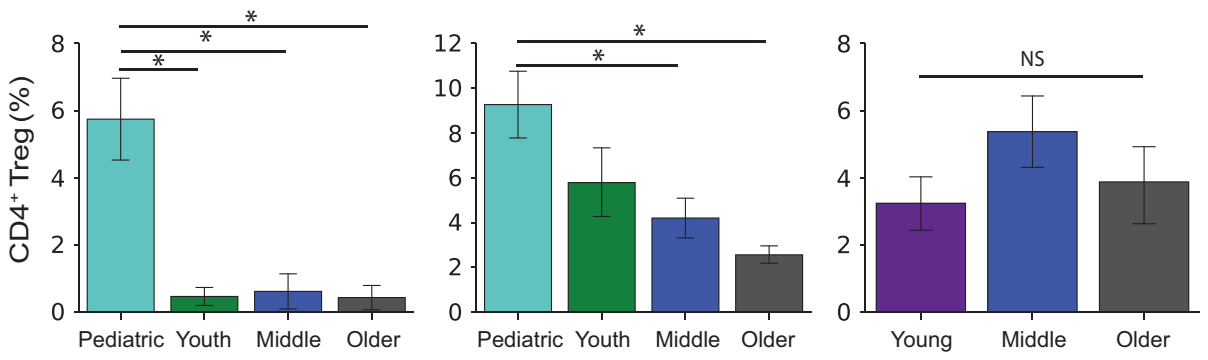

C
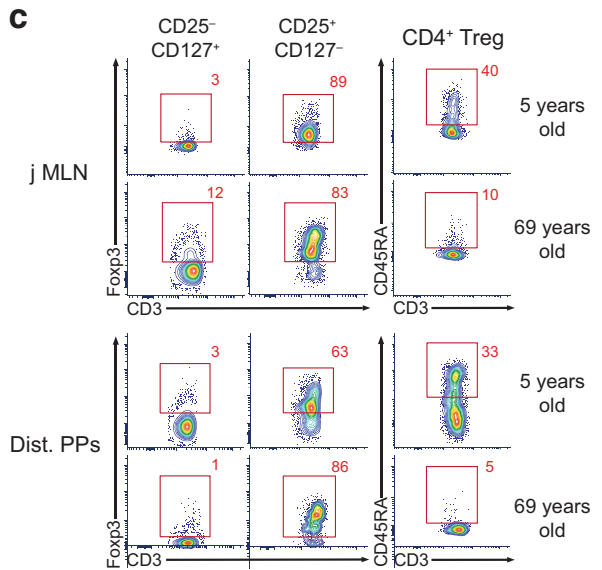

ILFs in colon
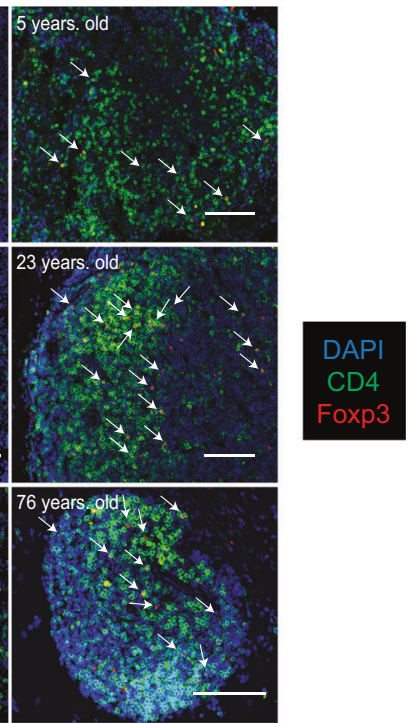

ILFs in colon d

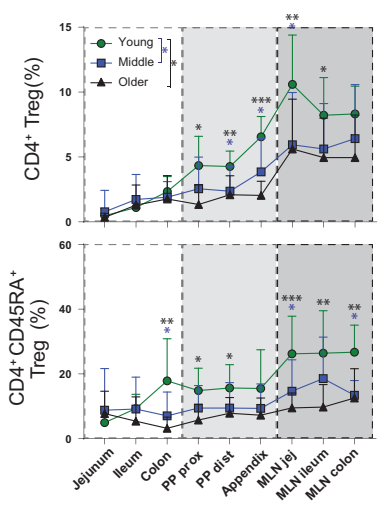

Fig. 5 Elevated frequency of Treg cells in lymphoid follicles, GALT, and $\mathrm{mLN}$ in younger donors. a Intestinal tissue sections were stained for CD4 (green), Foxp3 (red), and DAPI (blue). White arrows indicate CD4 ${ }^{+}$Foxp $3^{+}$cells with representative images from donors of indicated ages. Scale bars, $100 \mu \mathrm{m}$. b Quantification of Treg frequency from ILFs in jejunum (left; pediatric: $n=4$, avg. age 3 years; youth: $n=3$, avg. age 16 years; middle: $n=3$, avg. age 34 years; old: $n=3$, avg. age 70 years), PPs (center; pediatric: $n=3$, avg. age 5 years; youth: $n=4$, avg. age 18 years; middle: $n=4$, avg. age 38 years; older: $n=9$, avg. age 66 years), and ILFs in colon (right; young: $n=7$, avg. age 16 years; middle: $n=7$, avg. age 34 years; older: $n=5$, avg. age 66 years) using Imaris image software. ${ }^{* *}, p<0.01$. c Gating strategy for Treg cells shown from a representative jMLN of a young individual (Donor 339, 5 years) and from PP of an older individual (Donor 349, 69years), showing sequential gating on $\mathrm{CD} 25^{+} \mathrm{CD} 127^{-}$and Foxp3 expression, versus non-Treg $\left(\mathrm{CD} 25^{-} \mathrm{CD} 127^{+}\right)$cells. d Frequency (Mean $\pm \mathrm{SEM}$ ) of total (top) and CD45RA ${ }^{+}$ Treg (bottom) Tregs across the Gl tract from young (green, $n=10$ ), middle (blue; $n=13$ ), and older (gray; $n=13$ ) donors. Statistical significance comparing young to older (black*) and young to middle (blue ${ }^{*}$ ), indicated as ${ }^{*} p<0.05 ;{ }^{* *} p<0.01,{ }^{* * *} p<0.001$

within mucosal sites. We also reveal directionality in the ageassociated changes from jejunum to ileum and colon confirmed here by both imaging and flow cytometry, indicative of higher antigenic stimulation in small compared to large intestine.
The organized structures of the GALT and the draining $\mathrm{mLNs}$ are the main location for priming adaptive immune cell responses in the intestine. ${ }^{37}$ By imaging and flow cytometry analysis of PP from 55 donors, we show that PPs have numerous 


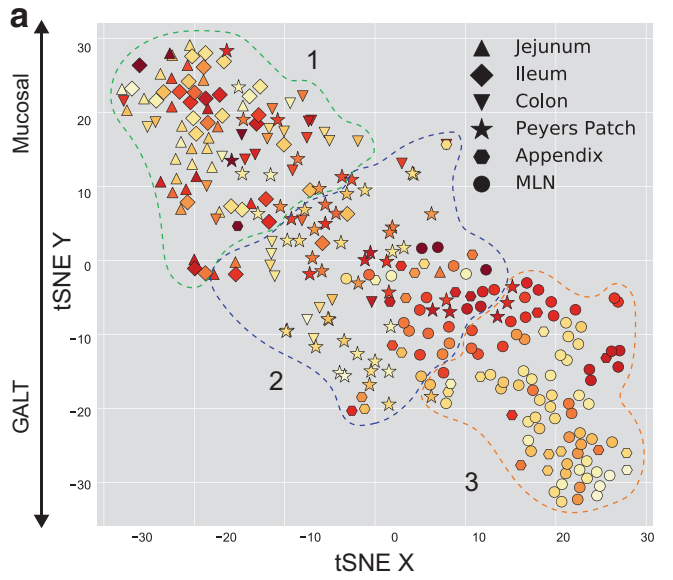

b
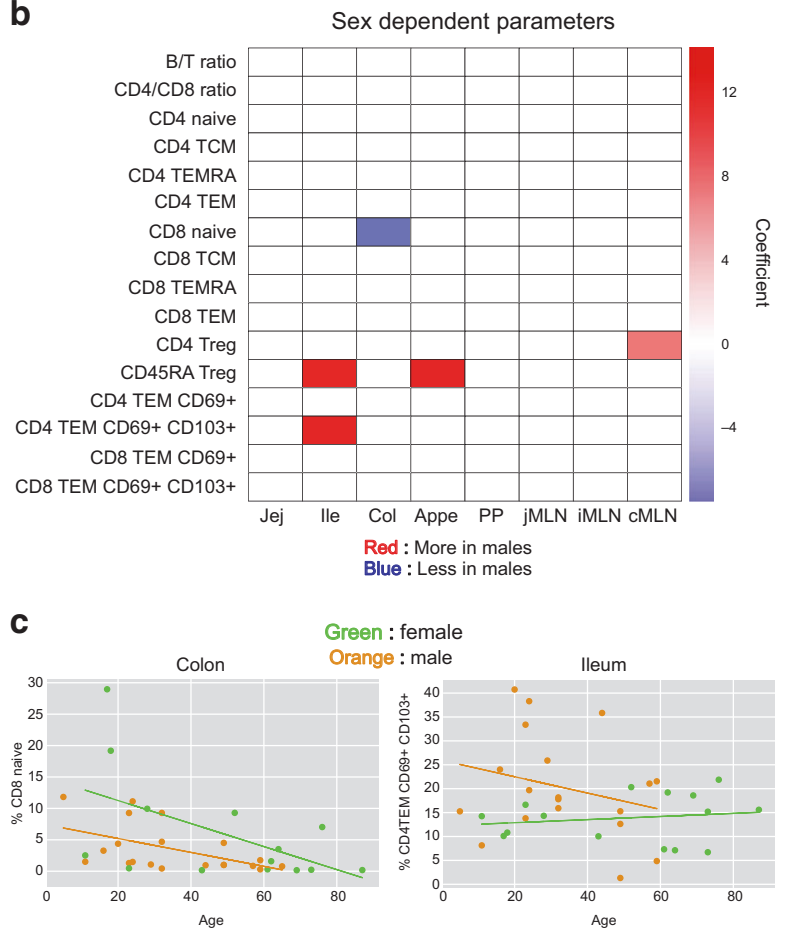
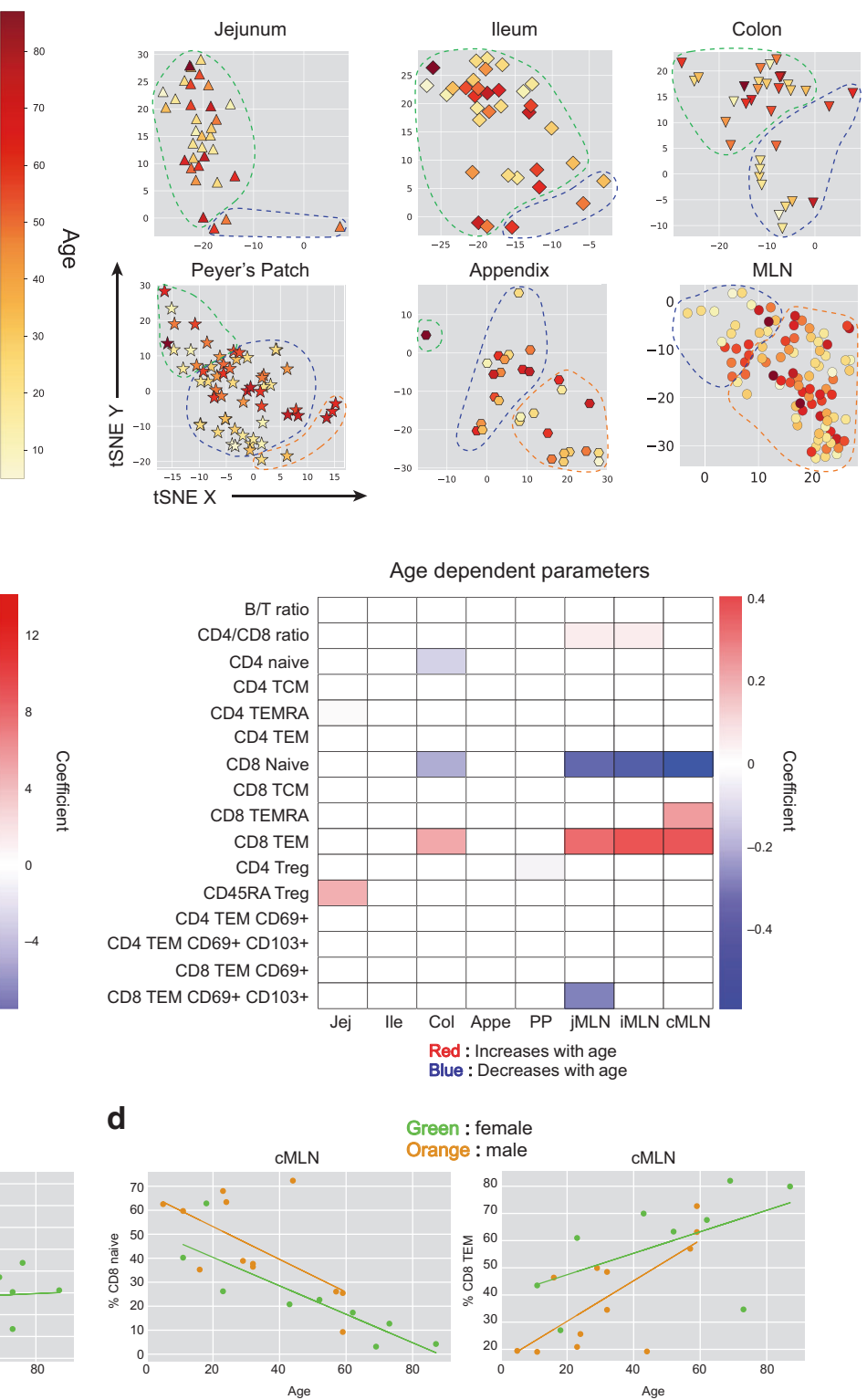

Fig. 6 Visualizing the effects of categorical variables on immunological parameters in the human Gl tract. a Graphical representation of dimensionality reduction of the dataset by $\mathrm{t}-\mathrm{SNE}$ (perplexity $=30$ ) for all flow cytometry data in the intestines, GALT, and mLN (left) and separated by each site (right). For all the plots, each symbol is an individual data point. The color bar represents a continuous scale from 0 to 89 years. Dashed lines mark the boundaries of k-means clusters identified. b Matrices show statistically significant associations between sex and immunological parameters measured adjusting for age (left) and statistically significant associations between age and immunological parameters measured adjusting for sex (right). White blocks indicate no significant relation between sex or age and the immunological parameter at the particular site. The color bar represents the coefficients for the main effect of sex (left) or age (right) on the immune parameter in each site, with darker red having a higher value in males (left) or with age (right) and darker blue having a lower value in males (left) or with age (right). Jej = Jejunum, Ile = lleum, Col = Colon, Appe = Appendix, PP = Peyer's Patch, jMLN= mesenteric lymph node of the jejunum, iMLN $=$ MLN of the ileum, and $c M L N=M L N$ of the colon. $\mathbf{c}$ Representative graphs showing sex-specific differences in naïve $C D 8^{+} T$ cell frequency in colon and CD4 ${ }^{+}$TRM frequency in ileum. $\mathbf{d}$ Representative graphs showing age-associated changes in naïve CD8 ${ }^{+} \mathrm{T}$ cell and CD8 ${ }^{+}$TEM frequency in the CMLN

$B$ cell follicles and interfollicular $T$ cells, which may reflect chronic stimulation by luminal antigen. We also demonstrate that the decline in naïve T cell frequency in PPs with age occurs more precipitously after age 40-50 years, consistent with our finding that active thymopoiesis may cease abruptly at some point after the age of $40 .{ }^{38}$ Interestingly, the proportion of naïve T cells in PPs after 50 years is very low, while a higher proportion of naïve $T$ cells are maintained even in advanced ages in adjoining $\mathrm{mLN}$. Therefore, naïve $\mathrm{T}$ cell replenishment in PP due to thymic output may be distinct from blood and lymph nodes, where naïve T cells are maintained by peripheral homeostasis. ${ }^{38,39}$

Our imaging data also reveal that intestinal Tregs are mostly localized in ILFs and PPs within the intestines, and are also present in $\mathrm{mLN}$. Treg frequency is highest in these sites in younger donors and declines significantly in middle and older years of adulthood. By contrast, TRM cells are the predominant $T$ cell subset within intestinal tissues, being localized in the LP and IEL regions, with fewer TRM cells (particularly the more mature $\mathrm{CD} 69^{+} \mathrm{CD} 103^{+} \mathrm{TRM}$ cells) in GALT and $\mathrm{mLN}$. These results suggest spatial and temporal 
a
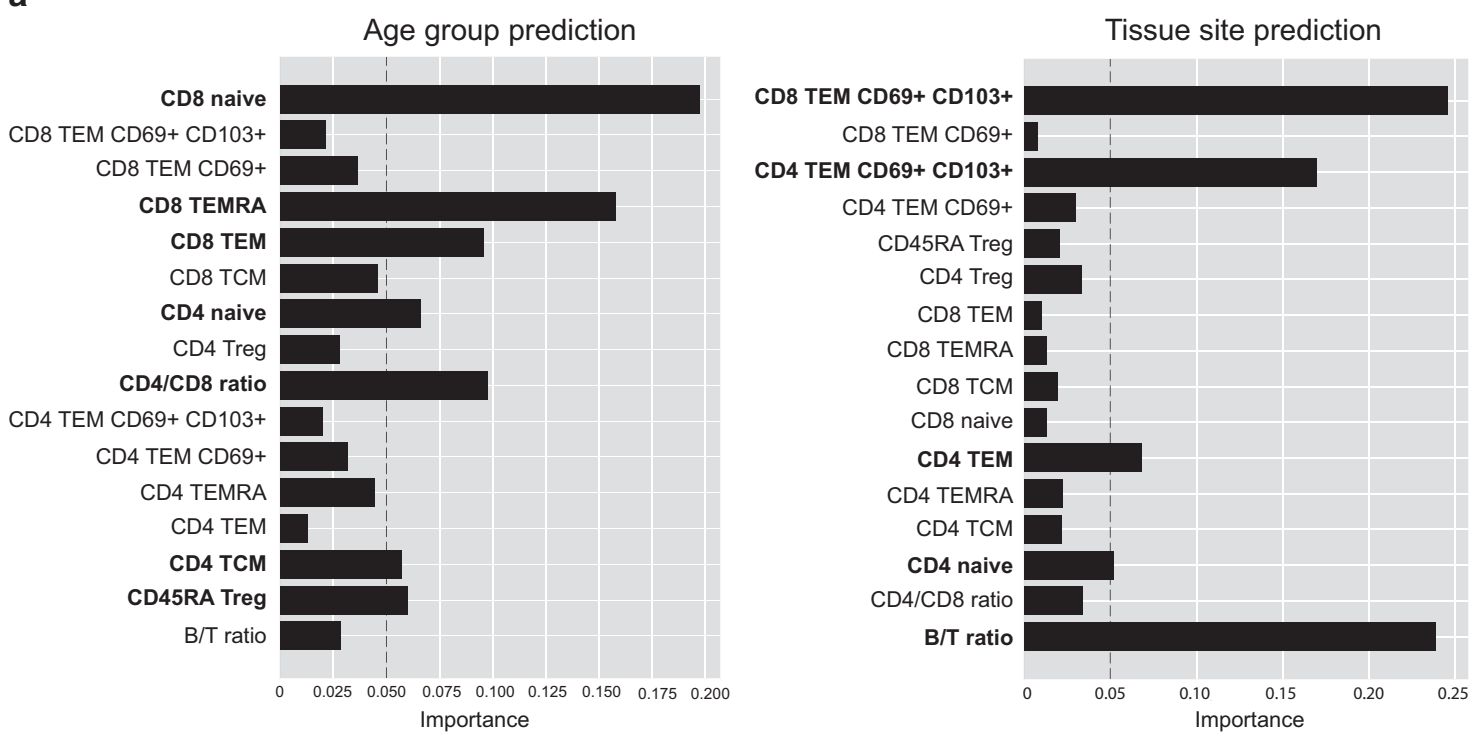

b

Age group prediction Accuracy score: 0.88

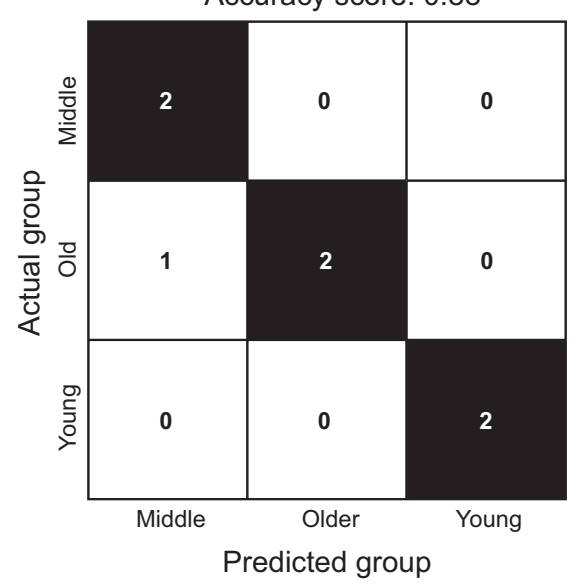

C

\begin{tabular}{|c|c|c|c|c|c|}
\hline & \multicolumn{5}{|c|}{$\begin{array}{l}\text { Tissue site prediction } \\
\text { Accuracy score: } 0.87\end{array}$} \\
\hline$\stackrel{2}{\stackrel{2}{\Sigma}}$ & 14 & 0 & 0 & 0 & 0 \\
\hline 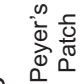 & 0 & 12 & 0 & 0 & 0 \\
\hline 응 & 0 & 2 & 9 & 0 & 0 \\
\hline$\stackrel{\underline{E}}{\underline{0}}$ & 0 & 1 & 1 & 4 & 1 \\
\hline : & 0 & 0 & 0 & 2 & 6 \\
\hline & MLN & $\begin{array}{c}\text { Peyer's } \\
\text { Patch }\end{array}$ & Colon & Ileum & Jejunum \\
\hline
\end{tabular}

Fig. 7 Machine learning models to predict age and tissue site in the human intestine based on immunological parameters. a Feature importance scores for Random forest classifier (RFc) used for predicting age group (left) and tissue site (right). The dotted black line indicates an importance score of 0.05 . The parameters selected to generate the final models are in bold. b Confusion matrix (CM) for predicted age groups from implementing the RFc on test dataset. $X$ axis is the predicted group and $Y$ axis is the actual age group of the sample. Numbers in each box of the CM indicate the number of samples from the test dataset predicted to fall in the particular age group. Correctly classified samples are along the diagonal of the CM (black blocks). c Confusion matrix (CM) for predicted tissue sites from implementing the RFc on test dataset. $X$ axis is the predicted tissue site and $Y$ axis is the actual tissue site of the sample. Numbers in each box of the $C M$ indicate the number samples of the test dataset predicted to fall in tissue site category. Correctly classified samples are along the diagonal of the CM (black blocks)

segregation of different critical functions of intestinal immunity; immune regulation by Tregs may occur specifically in follicular regions during childhood while maintenance of protective immunity by TRM occurs throughout mucosal tissues over life.

The age-associated changes in ILF content, naïve T cells and Tregs across the small intestine provide a cellular basis for the increased propensity for intestinal immunity and tolerance to develop in the early years. For example, oral vaccines such as those for polio or more recently rotavirus have proved particularly effective in infants and young children, ${ }^{40}$ consistent with increased ILFs with naïve $T$ cells providing enhanced targets for priming. Conversely, oral tolerance involving anergy or deletion of reactive $T$ cells and Treg induction ${ }^{41,42}$ is more readily achieved in children compared to adults in oral desensitization for milk allergy. ${ }^{43-45}$ It was also found that early exposure to peanuts during infancy promotes tolerance and avoids atopy. ${ }^{32}$ Mechanisms underlying increased Tregs in pediatric compared to adult donors are not known. In mice, tolerogenic DC (cDC1) can promote Treg induction, ${ }^{46}$ and we have identified increased $\mathrm{CDC} 1$ frequencies in the intestines of infants by flow cytometry ${ }^{22}$. We propose that the increased ILF content with distinct T and DC subsets in small intestines during early life promotes a highly regulated immune response to antigens introduced through the oral-Gl tract.

Our findings reveal a precise site- and age-determined $\mathrm{T}$ cell composition in the human Gl tract, defining immune signatures that could be used as a new baseline from which to define disease pathologies. Machine learning models have recently been applied for diagnostic purposes and are being developed for precision medicine. ${ }^{33-35}$ Here, we applied machine learning models to identify region- and age-related immune correlates of a healthy human intestine, revealing a highly correlative pattern of immune cell composition between regions of the intestine within a healthy individual. We propose that applying these immune parameters as 
a baseline will enable a more precise assessment of changes in the gut immune phenotype in different disease states.

In summary, we present here an anatomical analysis of the complex interaction of T cell immunity in the intestine, GALT and associated lymph nodes, revealing predictive quantitative changes that define tissue site and age. Our findings support an integral role for the $T$ cell component of adaptive immunity in human gut development, and in providing key protective and regulatory functions.

\section{METHODS}

Acquisition of tissue from human organ donors

Human tissues were obtained from deceased organ donors at the time of acquisition for life-saving clinical transplantation through an approved protocol and material transfer agreement with LiveOnNY. Donors were previously healthy and free of chronic disease, cancer, and were seronegative for Hepatitis B, C, and HIV. The study does not qualify as "human subjects" research, as confirmed by the Columbia University IRB as tissue samples were obtained from brain-dead (deceased) individuals.

Tissues preparation and lymphocyte isolation from human intestinal tissues

Tissue samples were maintained in cold saline or University of Wisconsin (UW) solution and brought to the laboratory within 2-4 $\mathrm{h}$ of organ procurement. Intestinal sections were opened along their mesenteric borders, washed in cold phosphate-buffered saline (PBS) (Mediatech, Manassas, VA). Jejunum was defined from the proximal two-fifths of the small intestine between the duodenojejunal flexure and ileocecal valve, and the proximal two-thirds of the ileum is the proximal ileum and the distal onethirds as the distal ileum, and colon samples were from the sigmoid colon. Proximal PPs were taken from the proximal ileum, distal PPs were from the distal ileum, and $\mathrm{mLNs}$ were separated from each regional mesentery. Samples were processed using enzymatic and mechanical digestion resulting in high yields of live lymphocytes, as described ${ }^{21}$.

Flow cytometry analysis

Single-cell suspensions were stained with the following fluorochrome-conjugated antibodies from Biolegned (San Diego, CA) for 30 min on ice: anti-human CD3 (OKT3), CD4 (SK3), CD8 (SK1), CD19 (HIB19), CD25 (BC96), CD45 (HI30), CD45RA (HI100), CD69 (FN50), CD103 (Ber-ACT8), CD127 (A019D5), and CCR7 (G043H7). Viability was determined using LIVE/DEAD fixable blue dead cell stain kit (Invitrogen, Carlsbad, CA). For intracellular staining, surface-stained cells were resuspended and incubated in $0.5 \mathrm{ml}$ fixation/permeabilization buffer (eBioscience), washed, resuspended in permeabilization buffer (eBioscience), and stained with anti-FOXP3 antibodies (236A/E7; eBioscience) and Ki67 (KI-67; BioLegend) for $60 \mathrm{~min}$ on ice and washed twice with permeabilization buffer. Stained cells were acquired on a 6-laser LSRII analytical flow cytometer (BD Biosciences) and analyzed using FCS Express 6 software (De Novo Software, Glendale, CA).

\section{Immunofluorescence imaging}

Jejunum, ileum including distal PPs, and colon samples were collected as described above, and were fixed immediately in $1.0 \%$ paraformaldehyde and 0.1 M L-lysine (Sigma-Aldrich, St. Louis, MO) add to PBS (pH7.4), incubated in $20 \%$ sucrose at $4{ }^{\circ} \mathrm{C}$, embedded in tissue-tek OCT compound (Sakura Finetek USA, Inc., Torrance, $\mathrm{CA}$ ), and stored at $-80^{\circ} \mathrm{C}$ until further use. Thin 5-8- $\mu \mathrm{m}$-thick sections were cut with a Leica CM 1950 cryostat (Leica Microsystems $\mathrm{GmbH}$, Wetzlar, Germany), permeabilized in PBS containing $0.1 \%$ Triton X-100 (Sigma-Aldrich) and 20\% Blocking One (Nacalai tesque, Kyoto, Japan), incubated in with fluorochrome-conjugated antibodies (anti-human CD4 (OKT4),
CD8 (HIT8a), CD45RA (HI100), Foxp3 (259D), and DAPI) in blocking buffer (PBS $+20 \%$ blocking reagent; Blocking One) at room temperature. Samples were mounted on coverslips with Prolong Diamond Antifade Mountant (Life Technologies, Inc., Gaithersburg, MD). Imaging was performed on an EVOS FL Auto 2 Imaging System (Invitrogen) with motorized $\mathrm{z}$ focus stage for fully automated image stitching. Specific cells were identified using the "spots" command in Imaris software (Bitplane, Zurich, Switzerland), and nonspecific fluorescence signals were manually removed. The LP lesion and ILFs area were defined using ImageJ software (National Institutes of Health, Bethesda, MD).

Statistical analysis and data visualization

Descriptive statistics (percent, mean, median, SEM) were calculated using GraphPad Prism (Graphpad softfware, San Diego, CA). Significant differences were assessed using a paired $t$-test, and correlations were assessed using linear regression. Multiple regression analysis of data was carried out using Python statsmodels module ${ }^{47}$. Ordinary least-squares (OLS) regression models were fitted for each of the immune parameters measured using age and sex as covariate. Coefficient and $p$-value was extracted from the OLS results table for the main effects of age and sex. The contribution of the main factor was considered significant in the models if $p$-value was $\leq 0.1$.

\section{t-SNE analysis of data}

Compiled flow cytometry data for CD4naive, CD4TCM, CD4TEMRA, CD4TEM, CD8naive, CD8TCM, CD8TEMRA, CD8TEM, CD4Treg, CD45RATreg, CD4TEM CD69 ${ }^{+}, \mathrm{CD}_{4}$ TEM CD69 ${ }^{+} \mathrm{CD} 103^{+}, \mathrm{CD} 8 \mathrm{TEM}$ $\mathrm{CD} 9^{+}, \mathrm{CD} 8 \mathrm{TEM} \mathrm{CD} 69^{+} \mathrm{CD}_{103^{+}}$frequency for all our donors as described in the sections above was used as an input for t-SNE analysis of the samples. The B:T and CD4:CD8 ratio values were excluded to avoid scaling bias. The analysis was run by implementing a custom Python script based on the sklearn. manifold.TSNE module. ${ }^{48}$ The perplexity was set to 30 and the model was run for 1000 iterations using the exact t-SNE method. The first two components of the t-SNE embedding were visualized using Python matplotlib library.

Random forest classifier training and prediction Random forest classifier was trained using Python Scikit-learn module. ${ }^{48}$ For predictions, immune parameters measured were averaged across all the sites for each donor to generate an individual immune signature, and subsequently classified into age groups. The resulting dataset was split into 80/20 train-test subsets which were used to train and test the classifier, respectively. For each of the models, 100-1000 trees were fitted during the training stage of model generation. To reduce the possibility of overfitting the models, feature selection was carried out by averaging the feature importance of each tree. The final model was generated to only include the top most contributing factors (Importance value $\geq 0.05$, Supplementary Figure $6 \mathrm{~b}$ and $\mathrm{c}$ ).

Accuracy score calculated as below was used to evaluate the different iterations of the trained model.

Accuracy score $=\left(\frac{n \text { correctly predicted test samples }}{N \text { total number of samples }}\right) \times 100$.

A confusion matrix was computed to evaluate the accuracy of a classification, such that in the matrix $X$, an observation $X_{i j}$ is equal to the number of observations in actual group $i$, predicted to be in group $j$. Values reported are prevalence-weighted macro-averages across classes.

\section{ACKNOWLEDGEMENTS}

This work was supported by NIH grants Al06697 and Al100119 awarded to D.L.F. P.D. was supported by a CRI-Irvington postdoctoral fellowship. Research reported here 
was performed in the CCTI Flow Cytometry Core, supported in part by the Office of the Director, National Institutes of Health under award S10RR027050. The content is solely the responsibility of the authors and does not necessarily represent the official views of the National Institutes of Health. We wish to gratefully acknowledge the generosity of the donor families and the exceptional efforts of LiveOnNY transplant coordinators and staff for making this study possible, and Dr Keita Okubo for help with donor tissue acquisition.

\section{AUTHOR CONTRIBUTIONS}

T.S. designed the experiments, obtained donor tissues, processed tissues, performed flow cytometry and tissue imaging experiments, collected the data, and wrote the paper; P.D. performed the statistical analysis, visualized the data, created machine learning models, and helped write the paper; D.J.C. obtained donor tissues; T.G, P.D. P.A.S., P.T., and M.M. processed tissues; K.F. assisted with tissue imaging experiments; D.L.F planned experiments, coordinated tissue acquisition and data acquisition/ analysis, analyzed data, and wrote the paper.

\section{ADDITIONAL INFORMATION}

The online version of this article (https://doi.org/10.1038/s41385-018-0110-8) contains supplementary material, which is available to authorized users.

Competing interests: The authors declare no competing interests.

\section{REFERENCES}

1. Gupta, R. S. et al. The prevalence, severity, and distribution of childhood food allergy in the United States. Pediatrics 128, e9-e17 (2011).

2. Bernstein, C. N. et al. The epidemiology of inflammatory bowel disease in Canada: a population-based study. Am. J. Gastroenterol. 101, 1559-1568 (2006).

3. Haggar, F. A. \& Boushey, R. P. Colorectal cancer epidemiology: incidence, mortality, survival, and risk factors. Clin. Colon Rectal Surg. 22, 191-197 (2009).

4. Hamada, $H$. et al. Identification of multiple isolated lymphoid follicles on the antimesenteric wall of the mouse small intestine. J. Immunol. 168, 57-64 (2002).

5. Peterson, L. W. \& Artis, D. Intestinal epithelial cells: regulators of barrier function and immune homeostasis. Nat. Rev. Immunol. 14, 141-153 (2014).

6. Pabst, O. et al. Cryptopatches and isolated lymphoid follicles: dynamic lymphoid tissues dispensable for the generation of intraepithelial lymphocytes. Eur. J. Immunol. 35, 98-107 (2005).

7. Gustafson, C. E. et al. Limited expression of APRIL and its receptors prior to intestinal IgA plasma cell development during human infancy. Mucosal Immunol. 7, 467-477 (2014).

8. Pearson, C., Uhlig, H. H. \& Powrie, F. Lymphoid microenvironments and innate lymphoid cells in the gut. Trends Immunol. 33, 289-296 (2012).

9. Chassaing, B. et al. Crohn disease-associated adherent-invasive E. coli bacteria target mouse and human Peyer's patches via long polar fimbriae. J. Clin. Invest. 121, 966-975 (2011).

10. Farstad, I. N., Halstensen, T. S., Fausa, O. \& Brandtzaeg, P. Heterogeneity of M-cellassociated B and T cells in human Peyer's patches. Immunology 83, 457-464 (1994).

11. Thome, J. J. C. et al. Spatial map of human $T$ cell compartmentalization and maintenance over decades of life. Cell 159, 814-828 (2014).

12. Masopust, D., Vezys, V., Wherry, E. J., Barber, D. L. \& Ahmed, R. Cutting edge: gut microenvironment promotes differentiation of a unique memory CD8 T cell population. J. Immunol. 176, 2079-2083 (2006).

13. Masopust, D. et al. Dynamic T cell migration program provides resident memory within intestinal epithelium. J. Exp. Med. 207, 553-564 (2010).

14. Kumar, B. V. et al. Human tissue-resident memory T cells are defined by core transcriptional and functional signatures in lymphoid and mucosal sites. Cell Rep. 20, 2921-2934 (2017).

15. Sakaguchi, S., Yamaguchi, T., Nomura, T. \& Ono, M. Regulatory T cells and immune tolerance. Cell 133, 775-787 (2008).

16. Coombes, J. L., Robinson, N. J., Maloy, K. J., Uhlig, H. H. \& Powrie, F. Regulatory T cells and intestinal homeostasis. Immunol. Rev. 204, 184-194 (2005).

17. Izcue, A., Coombes, J. L. \& Powrie, F. Regulatory lymphocytes and intestinal inflammation. Annu. Rev. Immunol. 27, 313-338 (2009).

18. Santiago, A. F. et al. Aging correlates with reduction in regulatory-type cytokines and T cells in the gut mucosa. Immunobiology 216, 1085-1093 (2011).

19. Carpenter, D. J. et al. Human immunology studies using organ donors: impact of clinical variations on immune parameters in tissues and circulation. Am. J. Transplant. 18, 74-88 (2018).
20. Thome, J. J. et al. Early-life compartmentalization of human T cell differentiation and regulatory function in mucosal and lymphoid tissues. Nat. Med. 22, 72-77 (2016).

21. Sathaliyawala, T. et al. Distribution and compartmentalization of human circulating and tissue-resident memory $T$ cell subsets. Immunity 38, 187-197 (2013).

22. Granot, T. et al. Dendritic cells display subset and tissue-specific maturation dynamics over human life. Immunity 46, 504-515 (2017).

23. Gordon, C. L. et al. Tissue reservoirs of antiviral T cell immunity in persistent human CMV infection. J. Exp. Med. 214, 651-667 (2017).

24. Cornes, J. S. Number, size, and distribution of Peyer's patches in the human small intestine: part I. The development of Peyer's patches. Gut 6, 225-229 (1965).

25. Lazuardi, L. et al. Age-related loss of naive T cells and dysregulation of T-cell/Bcell interactions in human lymph nodes. Immunology 114, 37-43 (2005).

26. Moghaddami, M., Cummins, A. \& Mayrhofer, G. Lymphocyte-filled villi: comparison with other lymphoid aggregations in the mucosa of the human small intestine. Gastroenterology 115, 1414-1425 (1998).

27. Sallusto, F., Geginat, J. \& Lanzavecchia, A. Central memory and effector memory $T$ cell subsets: function, generation, and maintenance. Annu. Rev. Immunol. 22, 745-763 (2004).

28. Sallusto, F., Lenig, D., Forster, R., Lipp, M. \& Lanzavecchia, A. Two subsets of memory $\mathrm{T}$ lymphocytes with distinct homing potentials and effector functions [see comments]. Nature 401, 708-712 (1999).

29. Laidlaw, B. J. et al. CD4+T cell help guides formation of CD103+lung-resident memory CD8+T cells during influenza viral infection. Immunity 41, 633-645 (2014).

30. Shiow, L. R. et al. CD69 acts downstream of interferon-alpha/beta to inhibit S1P1 and lymphocyte egress from lymphoid organs. Nature 440, 540-544 (2006).

31. Liu, W. et al. CD127 expression inversely correlates with FoxP3 and suppressive function of human CD4+T reg cells. J. Exp. Med. 203, 1701-1711 (2006).

32. Du Toit, G. et al. Randomized trial of peanut consumption in infants at risk for peanut allergy. N. Engl. J. Med. 372, 803-813 (2015).

33. Capper, D. et al. DNA methylation-based classification of central nervous system tumours. Nature 555, 469-474 (2018).

34. Mobadersany, P. et al. Predicting cancer outcomes from histology and genomics using convolutional networks. Proc. Natl. Acad. Sci. USA 115, E2970-E2979 (2018).

35. Poplin, R. et al. Prediction of cardiovascular risk factors from retinal fundus photographs via deep learning. Nat. Biomed. Eng. 2, 158-164 (2018).

36. Hovhannisyan, Z., Treatman, J., Littman, D. R. \& Mayer, L. Characterization of interleukin-17-producing regulatory $T$ cells in inflamed intestinal mucosa from patients with inflammatory bowel diseases. Gastroenterology 140, 957-965 (2011).

37. Stenstad, H. et al. Gut-associated lymphoid tissue-primed CD4+T cells display CCR9-dependent and -independent homing to the small intestine. Blood 107, 3447-3454 (2006).

38. Thome, J. J., et al. Longterm maintenance of human naive T cells through in situ homeostasis in lymphoid tissue sites. Sci. Immunol. 1, eaah6506 (2016).

39. den Braber, I. et al. Maintenance of peripheral naive T cells is sustained by thymus output in mice but not humans. Immunity 36, 288-297 (2012).

40. Halsey, N. \& Galazka, A. The efficacy of DPT and oral poliomyelitis immunization schedules initiated from birth to 12 weeks of age. Bull. World Health Organ 63, 1151-1169 (1985).

41. Dubois, B. et al. Sequential role of plasmacytoid dendritic cells and regulatory T cells in oral tolerance. Gastroenterology 137, 1019-1028 (2009).

42. Goubier, A. et al. Plasmacytoid dendritic cells mediate oral tolerance. Immunity 29, 464-475 (2008).

43. Morisset, $M$. et al. Oral desensitization in children with milk and egg allergies obtains recovery in a significant proportion of cases. A randomized study in 60 children with cow's milk allergy and 90 children with egg allergy. Eur. Ann. Allergy Clin. Immunol. 39, 12-19 (2007).

44. Longo, G. et al. Specific oral tolerance induction in children with very severe cow's milk-induced reactions. J. Allergy Clin. Immunol. 121, 343-347 (2008).

45. Skripak, J. M. et al. A randomized, double-blind, placebo-controlled study of milk oral immunotherapy for cow's milk allergy. J. Allergy Clin. Immunol. 122, 1154-1160 (2008).

46. Esterhazy, D. et al. Classical dendritic cells are required for dietary antigenmediated induction of peripheral T(reg) cells and tolerance. Nat. Immunol. 17, 545-555 (2016).

47. Statsmodels: Econometric and Statistical Modeling with Python. Proc. 9th Python Science Conf. (SCIPY 2010) (2010).

48. Pedregosa, F. et al. Scikit-learn: machine learning in Python. J. Mach. Learn. Res. 12, 2825-2830 (2011). 